\title{
A Generalization of Mahadevan's Version of the Krein-Rutman Theorem and Applications to $p$-Laplacian Boundary Value Problems
}

\author{
Yujun Cui ${ }^{1}$ and Jingxian Sun ${ }^{2}$ \\ ${ }^{1}$ Department of Applied Mathematics, Shandong University of Science and Technology, \\ Qingdao 266590, China \\ 2 Department of Mathematics, Xuzhou Normal University, Xuzhou 221116, China \\ Correspondence should be addressed to Yujun Cui, cyj720201@163.com
}

Received 12 January 2012; Revised 24 March 2012; Accepted 13 July 2012

Academic Editor: Lishan Liu

Copyright (C) 2012 Y. Cui and J. Sun. This is an open access article distributed under the Creative Commons Attribution License, which permits unrestricted use, distribution, and reproduction in any medium, provided the original work is properly cited.

\begin{abstract}
We will present a generalization of Mahadevan's version of the Krein-Rutman theorem for a compact, positively 1-homogeneous operator on a Banach space having the properties of being increasing with respect to a cone $P$ and such that there is a nonzero $u \in P \backslash\{\theta\}-P$ for which $M T^{p} u \geq$ $u$ for some positive constant $M$ and some positive integer $p$. Moreover, we give some new results on the uniqueness of positive eigenvalue with positive eigenfunction and computation of the fixed point index. As applications, the existence of positive solutions for $p$-Laplacian boundary-value problems is considered under some conditions concerning the positive eigenvalues corresponding to the relevant positively 1-homogeneous operators.
\end{abstract}

\section{Introduction}

The Krein-Rutman theorem [1,2] plays a very important role in nonlinear differential equations, as it provides the abstract basis for the proof of the existence of various principal eigenvalues, which in turn are crucial in bifurcation theory, in topological degree calculation, and in the stability analysis of solutions to elliptic equations. Owing to its importance, much attention has been given to the most general versions of the linear Krein-Rutman theorem by a number of authors, see [3-7]. For example, Krasnosel'skir [3] introduced the concept of the $e$-positive linear operator and then used it to prove the following results concerning the eigenvalues of positive linear compact operator.

Theorem 1.1. Let $X$, a Banach space, $P \subset X$ a cone in $X$. Let $T: X \rightarrow X$ be a linear, positive, and compact operator. Suppose that for some non-zero element $u=v-w$, where $v, w \in P$ and $-u \notin P$, 
the following relation is satisfied:

$$
M T^{p} u \geq u, \text { for some } M>0,
$$

where $p$ is some positive integer. Then $T$ has a non-zero eigenvector $x_{0}$ in $P$ :

$$
T x_{0}=\lambda_{0} x_{0}
$$

where the positive eigenvalue $\lambda_{0}$ satisfies the inequality $\lambda_{0} \sqrt[p]{M} \geq 1$.

Furthermore, if $P$ is a reproducing cone and $T$ is e-positive for some $e \in P \backslash\{\theta\}$, then

(1) the positive eigenvalue $\lambda_{0}$ of $T$ is simple;

(2) the operator $T$ has a unique positive eigenvector upto a multiplicative constant.

Recently, the nonlinear version of the Krein-Rutman theorem has been extended to positive eigenvalue problem for increasing, positively 1-homogeneous, compact, continuous operators by Mallet-Paret and Nussbaum [8,9], Mahadevan [10], and Chang [11].

The following nonlinear Krein-Rutman theorem has been established in [10].

Theorem 1.2. Let $X$ be a Banach space, $P \subset X$ be a cone in $X$. Let $T: X \rightarrow X$ be an increasing, positively 1-homogeneous, compact, continuous operator for which there exists a non-zero $u \in P$ and $M>0$ such that

$$
M T u \geq u ，
$$

Then Thas a non-zero eigenvector $x_{0}$ in $P$.

Compared with Theorem 1.1, we note that the element $u$, appeared in Theorem 1.2, belongs to $P$. Consequently we put forward a problem: are the results in Theorem 1.2 valid if the condition $u \in P$ is replaced with that in Theorem 1.1. The purpose of this study is to solve the above problem. By means of global structure of the positive solution set, we present a generalization of Mahadevan's version of the Krein-Rutman Theorem for a compact, positively 1-homogeneous operator on a Banach space having the properties of being increasing with respect to a convex cone $P$ and such that there is a non-zero $u \in$ $P \backslash\{\theta\}-P$ for which $M T^{p} u \geq u$ for some positive constant $M$ and some positive integer $p$. The method in this paper is somewhat different from that in [10].

The paper is organized as follow. In Section 2, we give some basic definitions and state three lemmas which are needed later. In Section 3, we establish some results for the existence of the eigenvalues of positively compact, 1-homogeneous operator and deduce some results on the uniqueness of positive eigenvalue with positive eigenfunction. In Section 4, we present some new methods of computation of the fixed point index for cone mapping. The final section is concerned with applications to the existence of positive solutions for $p$-Laplacian boundary-value problems under some conditions concerning the positive eigenvalues corresponding to the relevant positively 1-homogeneous operators. 


\section{Preliminaries}

Let $X$ a Banach space, $P \subset X$ be a cone in $X$. A cone $P$ is called solid if it contains interior points, that is, $\stackrel{\circ}{P} \neq \emptyset$. A cone is said to be reproducing if $X=P-P$. Every cone $P$ in $E$ defines a partial ordering in $E$ given by $x \leq y$ if and only if $y-x \in P$. If $x \leq y$ and $x \neq y$, we write $x<y$; if cone $P$ is solid and $y-x \in \stackrel{o}{P}$, we write $x \ll y$. For the concepts and the properties about the cone we refer to $[12,13]$.

A mapping $T: X \rightarrow X$ is said to be increasing if $x \leq y$ implies $T x \leq T y$ and it is said to be strictly increasing if $x<y$ implies $T x<T y$. The mapping is said to be compact if it takes bounded subsets of $X$ into relatively compact subsets of $X$. We say that the mapping is positively 1-homogeneous if it satisfies the relation

$$
T(t x)=t T(x) \quad \forall x \in X, t \in \mathbb{R}_{0}^{+}=[0,+\infty) .
$$

We say that a real number $\lambda$ is an eigenvalue of the operator if there exists a non-zero $x \in X$ such that $T x=\lambda x$.

Definition 2.1. Let $e \in P \backslash\{\theta\}$, a mapping $T: P \rightarrow P$ is called $e$-positive if for every non-zero $x \in P$ a natural number $n=n(x)$ and two positive number $c(x), d(x)$ can be found such that

$$
c(x) e \leq T^{n} x \leq d(x) e .
$$

This is stronger than requiring that $T$ is positive, that is, $T(P) \subset P$. It is always satisfied if $P$ is a solid cone and $T$ is strongly positive, that is, $T(P) \subset \stackrel{o}{P}$, with any $e \in P \backslash\{\theta\}$, but it can be satisfied more generally.

For the application in the sequel, we state the following three lemmas which can be found in [14, Theorem 17.1] [3, Lemma 1.2] [15, Theorem 1.1]. The first one involves the global structure of the positive solution set for completely continuous map, the second one involve cones, and the last one involves the computation of fixed-point index.

Lemma 2.2. Let $F: \mathbb{R}_{0}^{+} \times P \rightarrow P$ be a compact, continuous map and such that $F(0, x)=\theta$ for all $x \in P$. Then, $F(\lambda, x)=x$ has a nontrivial connected unbounded component of solutions $C^{+} \subset \mathbb{R}_{0}^{+} \times P$ containing the point $(0, \theta)$.

Lemma 2.3. Let $x \in P$. For an element $y \in X$, suppose a $\delta_{1}$ can be found such that $y \leq \delta_{1} x$. Then a small $\delta_{x}(y)$ exists for which $y \leq \delta_{x}(y) x$.

Lemma 2.4. Let $\Omega$ be a bounded open set in $X$, let $P$ be a cone in $X$, and let $A: P \rightarrow P$ be a completely continuous map. Suppose that there is an increasing, positively 1-homogeneous mapping $T$ and $u^{*} \in P \backslash\{\theta\}$ such that $T u^{*} \geq u^{*}$, and that

$$
A u \geq T u, \quad A u \neq u, \quad \forall u \in \partial \Omega \cap P .
$$

Then the fixed-point index $i(A, \Omega \cap P, P)=0$. 


\section{Main Results}

Theorem 3.1. Let $T: X \rightarrow X$ be an increasing, positively 1-homogeneous, compact, continuous mapping. Suppose that for some non-zero element $u=v-w$, where $v, w \in P$ and $-u \notin P$, the following relation is satisfied:

$$
M T^{p} u \geq u \quad(\text { for some } M>0),
$$

where $p$ is some positive integer. Then $T$ has a non-zero eigenvector $x_{0}$ in $P$ :

$$
T x_{0}=\lambda_{0} x_{0}
$$

where the positive eigenvalue $\lambda_{0}$ satisfies the inequality

$$
\lambda_{0} \sqrt[p]{M} \geq 1
$$

Proof. Let $v \in P(v \neq \theta)$ be as in the hypothesis of the theorem. For every positive integer $n>0$, define $F_{n}: \mathbb{R}_{0}^{+} \times P \rightarrow P$ by

$$
F_{n}(\lambda, x):=\lambda T x+\frac{1}{n} \lambda v
$$

Since $T$ is compact and continuous, each of these operators $F_{n}$ is clearly compact and continuous on $\mathbb{R}_{0}^{+} \times P$. Also they map $\mathbb{R}_{0}^{+} \times P$ into $P$ since $T$ maps $P$ into itself, which follows from the fact that $T$ is increasing and $T \theta=\theta$. Let, by Lemma 2.2, $C_{n}^{+} \subset \mathbb{R}_{0}^{+} \times P \rightarrow P$ be a connected unbounded branch of solutions to the equation

$$
F_{n}(\lambda, x)=x
$$

First we show that $C_{n}^{+} \subset[0, \sqrt[p]{M}] \times P$ for all $n>0$. Indeed, suppose that $x$ is a fixed point of $F_{n}(\lambda, \cdot)$ for some $\lambda \geq 1$. Then $x=F_{n}(\lambda, x)=\lambda T x+(1 / n) \lambda v$ and we obtain, from the properties of $T$ and the inequalities $v \geq \theta, v \geq u$, respectively, that

$$
x \geq \lambda T x, \quad x \geq \frac{1}{n} \lambda u .
$$

Let $\tau_{n}=\sup \{\tau \mid x \geq \tau u\}$. Obviously, $\tau_{n} \geq(1 / n) \lambda>0$. Since $T$ is increasing and 1homogeneous, by (3.4), we have

$$
\begin{aligned}
x & =\lambda T x+\frac{1}{n} \lambda v \geq \lambda^{2} T^{2} x+\frac{1}{n} \lambda v \\
& \geq \cdots \geq \lambda^{p} T^{p} x+\frac{1}{n} \lambda v \geq \lambda^{p} T^{p} x \\
& \geq \lambda^{p} T^{p}\left(\tau_{n} u\right) \geq \tau_{n} \frac{\lambda^{p}}{M} u .
\end{aligned}
$$


Consequently, by the definition of $\tau_{n}$,

$$
\frac{\lambda^{p}}{M} \leq 1
$$

In other words, if $\lambda>\sqrt[p]{M}$, then $F_{n}(\lambda, \cdot)$ has no fixed point. This implied that $C_{n}^{+} \subset[0, \sqrt[p]{M}] \times P$ for every $n>0$.

Notice that the branch $C_{n}^{+}$is connected and unbounded starting from $(0, \theta)$, there must necessarily exist $x_{n}$ with $\left\|x_{n}\right\|=1$ and $\lambda_{n} \in[0, \sqrt[p]{M}]$ such that $\left(\lambda_{n}, x_{n}\right) \in C_{n}^{+}$. That is,

$$
x_{n}=\lambda_{n} T x_{n}+\frac{1}{n} \lambda_{n} v, \quad \lambda_{n} \in[0, \sqrt[p]{M}],\left\|x_{n}\right\|=1 .
$$

Since the operator $T$ is compact, a subsequence of indices $n_{i}(i=1,2, \ldots)$ can be chosen such that the sequence $T x_{n}$ strongly converges to some element $y^{*} \in P$. By virtue of (3.9), with this choice of the sequence $n_{i}$, the convergence of the number $\lambda_{n_{i}}$ to some $\lambda_{*}$ which satisfies the inequality (3.3) can be guaranteed simultaneously. Then $x_{n_{i}}$ will converge in norm to the element $x_{0}=\lambda_{*} y^{*}$ with $\left\|x_{0}\right\|=1$. Further, it follows from the fact $\left\|x_{0}\right\|=1$ that $\lambda_{*} \neq 0$. Let $\lambda_{0}=\lambda_{*}^{-1}$. To obtain the equality (3.2), it suffices to pass to the limits in the equality:

$$
x_{n_{i}}=\lambda_{n_{i}} T x_{n_{i}}+\frac{1}{n_{i}} \lambda_{n_{i}} v \quad(i=1,2, \ldots)
$$

This completes the proof of the theorem.

Example 3.2. Consider the positive 1-homogeneous map T:

$$
(T x)(t)=\left(\int_{G} K(t, s) x^{p}(s) d s\right)^{1 / p},
$$

where $G$ is a bounded closed set in a finite-dimensional space, the kernel $K(t, s)$ is nonnegative, and $p=2 n+1$ for some $n \in \mathbb{N}$.

If there exists a system of points $s_{1}, s_{2}, \ldots, s_{p}$ such that

$$
K\left(s_{1}, s_{2}\right) K\left(s_{2}, s_{3}\right) \cdots K\left(s_{p}, s_{1}\right)>0 .
$$

Then the map $T$ defined by (3.11) has a nonnegative eigenfunction. In fact, it is easy to see that

$$
\left(T^{p} x\right)(t)=\left(\int_{G} K^{(p)}(t, s) x^{p}(s) d s\right)^{1 / p}
$$

where

$$
K^{(p)}(t, s)=\int_{G} \cdots \int_{G} K\left(t, t_{1}\right) \cdots K\left(t_{p-1}, s\right) d t_{1} \cdots d t_{p-1}
$$


is positive at the point $\left(s_{1}, s_{1}\right)$ of the topological product $G \times G$. We denoted by $G_{1} \subset G$ a closed neighborhood of the point $s_{1} \in G_{1}$ such that $K^{(p)}(t, s)>0$ when $t, s \in G_{1}$. We denote by $y(t)$ a continuous nonnegative function such that $y\left(s_{1}\right)>0, y(t)=0$ when $t \notin G_{1}$. Then

$$
\left(T^{p} y\right)(t)=\left(\int_{G} K^{(p)}(t, s) y^{p}(s) d s\right)^{1 / p}>0
$$

when $t \in G_{1}$. From (3.15) it follows that there exists a number $M>0$ such that

$$
M T^{p} y \geq y
$$

This inequality is just the condition of Theorem 3.1.

Remark 3.3. Positive 1-homogeneous maps are usually only defined on a cone. In this case, Theorem 3.1 remains valid provided $u \in P \backslash\{\theta\}$. Moreover, Theorem 2.1 and Corollary 2.1 of [5] already give a general result for a $k$-set contraction, positive 1-homogeneous maps.

Theorem 3.4. Suppose that $T$ is an increasing, positively 1-homogeneous, e-positive mapping. If there exist

$$
\begin{aligned}
& u_{1} \in P \backslash\{\theta\}, \lambda_{1}>0 \quad \text { such that } \lambda_{1} u_{1} \leq T u_{1}, \\
& u_{2} \in P \backslash\{\theta\}, \lambda_{2}>0 \quad \text { such that } \lambda_{2} u_{2} \geq T u_{2},
\end{aligned}
$$

then $\lambda_{2} \geq \lambda_{1}$. Furthermore, if for $x>y>\theta$, a positive number $c(x, y)$ can be found such that

$$
T x-T y \geq c(x, y) e
$$

then $\lambda_{1}=\lambda_{2}$ implies that $u_{1}$ is a scalar multiple of $u_{2}$.

Proof. It follows from the $e$-positiveness of $T$ that there exist $m, n$ such that

$$
T^{n} u_{1} \leq d\left(u_{1}\right) e, \quad c\left(u_{2}\right) e \leq T^{m} u_{2}, \quad\left(c\left(u_{1}\right), d\left(u_{2}\right)>0\right) .
$$

Then for $t>0$, we have

$$
\left(t c\left(u_{2}\right)-d\left(u_{1}\right)\right) e \leq t T^{m} u_{2}-T^{n} u_{1} \leq t \lambda_{2}^{m} u_{2}-\lambda_{1}^{n} u_{1}
$$

From this and the fact that $u_{1} \in P \backslash\{\theta\}$ we deduce that $\delta \triangleq \delta_{\lambda_{2}^{m} u_{2}}\left(\lambda_{1}^{n} u_{1}\right)>0$, that is, $\delta \lambda_{2}^{m} u_{2}-$ $\lambda_{1}^{n} u_{1} \in P$. Since $T$ is increasing, $T\left(\delta \lambda_{2}^{m} u_{2}\right) \geq T\left(\lambda_{1}^{n} u_{1}\right)$, from which, by virtue of (3.17), it follows that

$$
\lambda_{2} \delta \lambda_{2}^{m} u_{2} \geq T\left(\delta \lambda_{2}^{m} u_{2}\right) \geq T\left(\lambda_{1}^{n} u_{1}\right) \geq \lambda_{1} \lambda_{1}^{n} u_{1}
$$

By Lemma 2.3, we obtain that $\lambda_{2} \geq \lambda_{1}$. 
Now suppose that $\lambda_{1}=\lambda_{2}$. According to the above proof, the number $\delta>0$. Since $\delta \lambda_{2}^{m} u_{2}-\lambda_{1}^{n} u_{1} \in P$ and (3.18), if $\delta \lambda_{2}^{m} u_{2} \neq \lambda_{1}^{n} u_{1}$, then there exists $c>0$ such that

$$
T\left(\delta \lambda_{2}^{m} u_{2}\right)-T\left(\lambda_{1}^{n} u_{1}\right) \geq c e .
$$

Therefore, by (3.19)

$$
\begin{aligned}
\lambda_{2} \delta \lambda_{2}^{m} u_{2} & \geq T\left(\delta \lambda_{2}^{m} u_{2}\right) \geq T\left(\lambda_{1}^{n} u_{1}\right)+c e \geq \lambda_{1} \lambda_{1}^{n} u_{1}+\frac{c}{d\left(u_{1}\right)} T^{n}\left(u_{1}\right) \\
& \geq \lambda_{1}^{n+1} u_{1}+\frac{c}{d\left(u_{1}\right)} \lambda_{1}^{n} u_{1}=\left(1+\frac{c}{\lambda_{1} d\left(u_{1}\right)}\right) \lambda_{2} \lambda_{1}^{n} u_{1} .
\end{aligned}
$$

This contradicts with the definition of $\delta$. This shows that we must have $\delta \lambda_{2}^{m} u_{2}=\lambda_{1}^{n} u_{1}$. This completes the proof of the theorem.

\section{Computation for the Fixed-Point Index}

We illustrate how $e$-positivity can be used to prove some fixed-point index results which can then be used to prove existence results for nonlinear equations. When $\Omega$ is a bounded open set in a Banach space $X$, we write $\Omega_{P}:=\Omega \cap P$ and $\partial \Omega_{P}$ for its boundary relative to $P$.

Theorem 4.1. Let $\Omega$ be a bounded open set in $X$ containing $\theta$, let $P$ be a cone in $X$, and let $A: P \rightarrow P$ be a completely continuous map. Suppose that there is an increasing, positively 1-homogeneous, $e$ positive mapping $T$ such that $T e \leq e$, and that

$$
A u \leq T u, \quad A u \neq u \quad \forall u \in \partial \Omega_{P} .
$$

Then the fixed-point index $i\left(A, \Omega_{P}, P\right)=1$.

Proof. We show that $A u \neq \mu u$ for all $u \in \partial \Omega_{P}$ and all $\mu \geq 1$, from which the result follows by standard properties of fixed-point index (see, e.g., [12-14]). Suppose that there exist $u_{0} \in \partial \Omega_{P}$ and $\mu_{0} \geq 1$ such that $A u_{0}=\mu_{0} u_{0}$, then $\mu_{0}>1$. It follows from the $e$-positiveness of $T$ that there exists a natural number $n$ such that

$$
T^{n} u_{0} \leq d\left(u_{0}\right) e, d\left(u_{0}\right)>0
$$

So, by induction, for all $m \in N$, we have

$$
\mu_{0}^{m n} u_{0} \leq T^{m n} u_{0} \leq d\left(u_{0}\right) e
$$

which implies $u_{0}=\theta$. This contradicts $u_{0} \in \partial \Omega_{P}$. 
Theorem 4.2. Let $P$ be a normal cone in a real Banach space $X$ and let $A: P \rightarrow P$ be a completely continuous map. Suppose that there is an increasing, positively 1-homogeneous, e-positive mapping $T$ (with $n=1$ in Definition 2.1) which satisfies the following conditions:

(1) there exists $k \in[0,1)$ such that

$$
\text { Te } \leq k e,
$$

(2) there exists $M>0$ such that

$$
A u \leq T u+M e, \quad u \in P .
$$

Then there exists $R_{1}>0$ such that for any $R>R_{1}$, the fixed-point index $i\left(A, B_{R} \cap P, P\right)=1$, where $B_{R}=\{x \in X \mid\|x\| \leq R\}$.

Proof. Let

$$
W=\{u \in P \mid u=\mu A u, 0 \leq \mu \leq 1\} .
$$

In the following, we prove that $W$ is bounded.

For any $u \in W \backslash\{\theta\}$, using the $e$-positiveness of $T$, we have

$$
u=\mu A u \leq T u+M e \leq(d(u)+M) e .
$$

Let

$$
\mu_{0}=\inf \{\mu \mid u \leq \mu e\}
$$

It is easy to see that $0<\mu_{0}<+\infty$ and $u \leq \mu_{0} e$. We now have

$$
u \leq T u+M e \leq\left(k \mu_{0}+M\right) e,
$$

which, by the definition of $\mu_{0}$, implies that $\mu_{0} \leq M /(1-k)$. So we know that $u \leq(M /(1-k)) e$ and $W$ is bounded by the normality of the cone $P$.

Select $R_{1}>\sup \{\|x\| \mid x \in W\}$. Then from the homotopy invariance property of fixedpoint index we have

$$
i\left(A, B_{R} \cap P, P\right)=i\left(\theta, B_{R} \cap P, P\right)=1, \quad \forall R>R_{1} .
$$

This completes the proof of the theorem. 


\section{Applications}

In the following, we will apply the results in this paper to the existence of positive solution for two-point boundary-value problems for one-dimensional $p$-Laplacian:

$$
\begin{gathered}
\left(\phi_{p}\left(v^{\prime}\right)\right)^{\prime}+a(t) f(v(t))=0, \quad t \in(0,1), \\
v^{\prime}(0)=0, \quad v(1)=0,
\end{gathered}
$$

where $\phi_{p}(s)=|s|^{p-2} s, p \geq 2$, and $\left(\phi_{p}\right)^{-1}=\phi_{q}=|s|^{q-2} s,(1 / p)+(1 / q)=1$.

We make the following assumptions:

$\left(H_{1}\right) f:[0,+\infty) \rightarrow[0,+\infty)$ is continuous;

$\left(H_{2}\right) a:[0,1] \rightarrow(0,+\infty)$ is continuous.

For each $v \in E:=C[0,1]$, we write $\|v\|=\max \{|v(t)|: t \in[0,1]\}$. Define

$$
K=\{v \in E: v(t) \geq(1-t)\|v\|\}
$$

Clearly, $(E,\|\cdot\|)$ is a Banach space and $K$ is a cone of $E$. For any real constant $r>0$, define $B_{r}=\{v \in E:\|x\|<r\}$.

Let the operators $T$ and $A$ be defined by:

$$
\begin{aligned}
& (T v)(t)=\int_{t}^{1} \phi_{q}\left(\int_{0}^{s} a(\tau) v^{p-1}(\tau) d \tau\right) d s, \\
& (A v)(t)=\int_{t}^{1} \phi_{q}\left(\int_{0}^{s} a(\tau) f(v(\tau)) d \tau\right) d s
\end{aligned}
$$

respectively.

Under $\left(H_{1}\right)$ and $\left(H_{2}\right)$, it is not difficult to verify that the non-zero fixed points of the operator $A$ are the positive solutions of boundary-value problem (5.1). In addition, we have from $\left(H_{2}\right)$ that $T: K \rightarrow E$ is a completely continuous, positively 1-homogeneous operator and $T(K) \subset K$.

Lemma 5.1. Suppose that $\left(\mathrm{H}_{2}\right)$ holds. Then for the operator $T$ defined by (5.3), there is a unique positive eigenvalue $\lambda_{1}$ of $T$ with its eigenfunction in $K$.

Proof. First, we show that $T$ is $e$-positive with $e=1-t$, that is, for any $v>\theta$ from $K$, there exist $\alpha, \beta>0$ such that

$$
\alpha e \leq T v \leq \beta e .
$$

Let $M_{1}=\max _{t \in[0,1]} a(t)$. Then

$$
(T v)(t) \leq \int_{t}^{1} \phi_{q}\left(M_{1}\|v\|^{p-1}\right) d s=\phi_{q}\left(M_{1}\|v\|^{p-1}\right)(1-t) .
$$

So, we may take $\beta=\phi_{q}\left(M_{1}\|v\|^{p-1}\right)$. 
Clearly, we may take $\alpha=\|T v\|=(T v)(0)$ since $T(K) \subset K$. So (5.4) is proved.

Now we need to show that for any $u>v>\theta$, there always exists some $c>0$ such that

$$
T u-T v \geq c e .
$$

In fact, we note that $\phi_{q}$ is increasing, there exists an $\eta \in(0,1)$ such that

$$
m=\min _{s \in[\eta, 1]} \phi_{q}\left(\int_{0}^{s} a(\tau) u^{p-1}(\tau) d \tau\right)-\phi_{q}\left(\int_{0}^{s} a(\tau) v^{p-1}(\tau) d \tau\right)>0
$$

Then for all $t \in[\eta, 1]$, we have

$$
\begin{aligned}
(T u)(t)-(T v)(t) \geq & \int_{\eta}^{1} \phi_{q}\left(\int_{0}^{s} a(\tau) u^{p-1}(\tau) d \tau\right) d s \\
& -\int_{\eta}^{1} \phi_{q}\left(\int_{0}^{s} a(\tau) v^{p-1}(\tau) d \tau\right) d s \geq m(1-t) .
\end{aligned}
$$

Since $(T u)(t)-(T v)(t) \geq(T u)(\eta)-(T v)(\eta) \geq m(1-\eta)$ for all $t \in[0, \eta]$, we have

$$
(T u)(t)-(T v)(t) \geq m(1-\eta)(1-t)
$$

for all $t \in[0,1]$. Therefore, the proof is complete and follows from Theorems 3.1 and 3.4.

Remark 5.2. Let $\varphi^{*}$ be the positive eigenfunction of $T$ corresponding to $\lambda_{1}$, thus $\lambda_{1} T \varphi^{*}=\varphi^{*}$. Then by Lemma 5.1 , there exist $\alpha, \beta>0$ such that

$$
\alpha e \leq T \varphi^{*}=\lambda_{1} \varphi^{*} \leq \beta e .
$$

Hence we obtained that $T$ is $\varphi^{*}$-positive operator.

Theorem 5.3. Suppose that the conditions $\left(H_{1}\right)$ and $\left(H_{2}\right)$ are satisfied, and

$$
\begin{gathered}
\liminf _{u \rightarrow 0^{+}} \frac{f(u)}{u^{p-1}}>\lambda_{1}^{1-p}, \\
\limsup _{u \rightarrow+\infty} \frac{f(u)}{u^{p-1}}<\lambda_{1}^{1-p},
\end{gathered}
$$

where $\lambda_{1}$ is given in Lemma 5.1, then the boundary-value problem (5.1) has at least one positive solution. 
Proof. It follows from (5.11) that there exists $r>0$ such that

$$
f(u) \geq \lambda_{1}^{1-p} u^{p-1}, \quad \forall 0 \leq u \leq r .
$$

We may suppose that $A$ has no fixed point on $\partial B_{r} \cap K$ (otherwise, the proof is finished). Therefore by (5.13),

$$
(A v)(t) \geq \int_{t}^{1} \phi_{q}\left(\int_{0}^{s} a(\tau) \lambda_{1}^{1-p} u^{p-1}(\tau) d \tau\right) d s=\frac{1}{\lambda_{1}}(T v)(t), \quad v \in \partial B_{r} \cap K
$$

Hence we have from Lemma 2.4 and Remark 5.2 that

$$
i\left(A, B_{r} \cap K, K\right)=0 .
$$

It follows from (5.12) that there exist $0<\sigma<1$ and $M_{1}>0$ such that

$$
f(u) \leq \sigma^{p-1} \lambda_{1}^{1-p} u^{p-1}+M_{2}, \quad \forall u \geq 0 .
$$

Thus, we have

$$
\begin{aligned}
(A v)(t) & \leq \int_{t}^{1} \phi_{q}\left(\int_{0}^{s} a(\tau)\left[\sigma^{p-1} \lambda_{1}^{1-p} u^{p-1}(\tau)+M_{2}\right] d \tau\right) d s \\
& \leq \frac{\sigma}{\lambda_{1}} \int_{t}^{1} \phi_{q}\left(\int_{0}^{s} a(\tau) u^{p-1}(\tau) d \tau\right) d s+\int_{t}^{1} \phi_{q}\left(M_{1} M_{2}\right) d s \\
& =\frac{\sigma}{\lambda_{1}}(T v)(t)+\phi_{q}\left(M_{1} M_{2}\right)(1-t), \quad v \in K .
\end{aligned}
$$

Here we have used the following inequality:

$$
\phi_{q}(a+b) \leq \phi_{q}(a)+\phi_{q}(b), \quad a, b>0,1<q \leq 2 .
$$

Thus by Theorem 4.2 and Remark 5.2, there exists $R_{1}>r$ such that

$$
i\left(A, B_{R} \cap K, K\right)=0, \quad R>R_{1},
$$


and hence we obtained

$$
i\left(A,\left(B_{R} \backslash \overline{B_{r}}\right) \cap K, K\right)=1 .
$$

Thus, $A$ has a fixed point in $\left(B_{R} \backslash \overline{B_{r}}\right) \cap K$. Consequently, (5.1) has a positive solution.

Theorem 5.4. Suppose that the conditions $\left(H_{1}\right)$ and $\left(H_{2}\right)$ are satisfied, and

$$
\begin{aligned}
& \limsup _{u \rightarrow 0^{+}} \frac{f(u)}{u^{p-1}}<\lambda_{1}^{1-p}, \\
& \liminf _{u \rightarrow+\infty} \frac{f(u)}{u^{p-1}}>\lambda_{1}^{1-p},
\end{aligned}
$$

where $\lambda_{1}$ is given in Lemma 5.1, then the boundary-value problem (5.1) has at least one positive solution.

Proof. It follows from (5.21) that there exists $r_{2}>0$ such that

$$
f(u) \leq \lambda_{1}^{1-p} u^{p-1}, \quad \forall 0 \leq u \leq r_{2}
$$

We may suppose that $A$ has no fixed point on $\partial B_{r_{2}} \cap K$ (otherwise, the proof is finished). Therefore by (5.23),

$$
(A v)(t) \leq \int_{t}^{1} \phi_{q}\left(\int_{0}^{s} a(\tau) \lambda_{1}^{1-p} u^{p-1}(\tau) d \tau\right) d s=\frac{1}{\lambda_{1}}(T v)(t), \quad v \in \partial B_{r_{2}} \cap K
$$

Hence we have from Theorem 4.1 and Remark 5.2 that

$$
i\left(A, B_{r_{2}} \cap K, K\right)=1
$$

It follows from (5.22) that there exists $\varepsilon>0$ such that $f(u) \geq\left(\lambda_{1}^{1-p}+\varepsilon\right) u^{p-1}$ when $u$ is sufficiently large. We know from the continuity of $f$ that there exists $b \geq 0$ such that

$$
f(u) \geq\left(\lambda_{1}^{1-p}+\varepsilon\right) u^{p-1}-b, \quad \forall u \geq 0 .
$$

Take

$$
R_{2}>\max \left\{r_{2},\left(\frac{M_{1} b 2^{p}}{m_{0} \varepsilon}\right)^{1 /(p-1)}\right\}
$$

where $m_{0}=\min _{t \in[0,1]} a(t), M_{1}=\max _{t \in[0,1]} a(t)$. 
For $v \in \partial B_{R_{2}} \cap K$, we have

$$
\begin{aligned}
\int_{0}^{s} a(\tau) f(v(\tau)) d \tau & \geq \int_{0}^{s} a(\tau)\left[\left(\lambda_{1}^{1-p}+\varepsilon\right) v^{p-1}(\tau)-b\right] d \tau \\
& \geq \lambda_{1}^{1-p} \int_{0}^{s} a(\tau) v^{p-1}(\tau) d \tau+m_{0} \varepsilon \int_{0}^{s}(1-\tau)^{p-1}\|v\|^{p-1} d \tau-M_{1} b s \\
& =\lambda_{1}^{1-p} \int_{0}^{s} a(\tau) v^{p-1}(\tau) d \tau+m_{0} \varepsilon R_{2}^{p-1} \int_{0}^{s}(1-\tau)^{p-1} d \tau-M_{1} b s \\
& \geq \lambda_{1}^{1-p} \int_{0}^{s} a(\tau) v^{p-1}(\tau) d \tau+m_{0} \varepsilon R_{2}^{p-1} \int_{0}^{\min \{s, 1 / 2\}}(1-\tau)^{p-1} d \tau-M_{1} b s \\
& \geq \lambda_{1}^{1-p} \int_{0}^{s} a(\tau) v^{p-1}(\tau) d \tau+m_{0} \varepsilon R_{2}^{p-1} \int_{0}^{\min \{s, 1 / 2\}}\left(1-\frac{1}{2}\right)^{p-1} d \tau-M_{1} b s \\
& \geq \lambda_{1}^{1-p} \int_{0}^{s} a(\tau) v^{p-1}(\tau) d \tau+\left(m_{0} \varepsilon R_{2}^{p-1} \frac{1}{2^{p}}-M_{1} b\right) s \\
& \geq \lambda_{1}^{1-p} \int_{0}^{s} a(\tau) v^{p-1}(\tau) d \tau .
\end{aligned}
$$

Thus, we have

$$
(A v)(t) \geq \int_{t}^{1} \phi_{q}\left(\int_{0}^{s} a(\tau) \lambda_{1}^{1-p} u^{p-1}(\tau) d \tau\right) d s=\frac{1}{\lambda_{1}}(T v)(t), \quad v \in \partial B_{R_{2}} \cap K .
$$

It follows from Lemma 2.4 that

$$
i\left(A, B_{R_{2}} \cap K, K\right)=0,
$$

and hence we obtained

$$
i\left(A,\left(B_{R_{2}} \backslash \overline{B_{r_{2}}}\right) \cap K, K\right)=-1 .
$$

Thus, $A$ has a fixed point in $\left(B_{R_{2}} \backslash \overline{B_{r_{2}}}\right) \cap K$. Consequently, (5.1) has a positive solution.

Remark 5.5. $p$-Laplacian boundary-value problems have been studied by some authors $([16,17]$ and references therein). In preceding works mentioned, they study the existence of positive solutions by the shooting method, fixed-point theorem, or the fixed-point index under some different conditions. It is known that, when $p=2$, there are very good conditions imposed on $f$ that ensure the existence of positive solution for two-point boundary-value problems (5.1). In particular, some of those involving the first eigenvalues corresponding to the relevant linear operator are sharp conditions. So, Theorems 5.3 and 5.4 generalize a number of recent works about the existence of solutions for $p$-Laplacian boundary-value problems. 


\section{Acknowledgments}

The authors sincerely thank the reviewers for their valuable suggestions and useful comments that have led to the present improved version of the original paper. The paper is supported by the National Science Foundation of China (10971179) and Research Award Fund for Outstanding Young Scientists of Shandong Province (BS2010SF023).

\section{References}

[1] M. G. Krĕn and M. A. Rutman, "Linear operators leaving invariant a cone in a Banach space," Uspekhi Matematicheskikh Nauk, vol. 23, no. 1, pp. 3-95, 1948 (Russian), English translation: American Mathematical Society Translations, vol. 26, 1950.

[2] M. G. Kreı̆n and M. A. Rutman, "Linear operators leaving invariant a cone in a Banach space," American Mathematical Society Translations, vol. 10, pp. 199-325, 1962.

[3] M. A. Krasnosel'skiॅ, Positive Solutions of Operator Equations, Translated from the Russian by R. E. Flaherty, edited by L. F. Boron, P. Noordhoff Ltd., Groningen, The Netherlands, 1964.

[4] M. A. Krasnosel'skii, Topological Methods in the Theory of Nonlinear Integral Equations, The Macmillan, New York, NY, USA, 1964.

[5] R. D. Nussbaum, "Eigenvectors of nonlinear positive operators and the linear Kreln-Rutman theorem," in Fixed Point Theory, vol. 886 of Lecture Notes in Mathematics, pp. 309-330, Springer, Berlin, Germany, 1981.

[6] R. D. Nussbaum, "Eigenvectors of order-preserving linear operators," Journal of the London Mathematical Society Second Series, vol. 58, no. 2, pp. 480-496, 1998.

[7] J. R. L. Webb, "Remarks on $u_{0}$-positive operators," Journal of Fixed Point Theory and Applications, vol. 5, no. 1, pp. 37-45, 2009.

[8] J. Mallet-Paret and R. D. Nussbaum, "Eigenvalues for a class of homogeneous cone maps arising from max-plus operators," Discrete and Continuous Dynamical Systems Series A, vol. 8, no. 3, pp. 519-562, 2002.

[9] J. Mallet-Paret and R. D. Nussbaum, "Generalizing the Krein-Rutman theorem, measures of noncompactness and the fixed point index," Journal of Fixed Point Theory and Applications, vol. 7, no. 1, pp. 103-143, 2010.

[10] R. Mahadevan, "A note on a non-linear Krein-Rutman theorem," Nonlinear Analysis: Theory, Methods $\mathcal{E}$ Applications, vol. 67, no. 11, pp. 3084-3090, 2007.

[11] K. C. Chang, "A nonlinear Krein Rutman theorem," Journal of Systems Science E Complexity, vol. 22, no. 4, pp. 542-554, 2009.

[12] K. Deimling, Nonlinear Functional Analysis, Springer, Berlin, Germany, 1985.

[13] D. J. Guo and V. Lakshmikantham, Nonlinear Problems in Abstract Cones, vol. 5 of Notes and Reports in Mathematics in Science and Engineering, Academic Press, Boston, Mass, USA, 1988.

[14] H. Amann, "Fixed point equations and nonlinear eigenvalue problems in ordered Banach spaces," SIAM Review, vol. 18, no. 4, pp. 620-709, 1976.

[15] S. Jingxian and L. Xiaoying, "Computation for topological degree and its applications," Journal of Mathematical Analysis and Applications, vol. 202, no. 3, pp. 785-796, 1996.

[16] F.-H. Wong, "Existence of positive solutions for $m$-Laplacian boundary value problems," Applied Mathematics Letters, vol. 12, no. 3, pp. 11-17, 1999.

[17] B. Liu, "Positive solutions of singular three-point boundary value problems for the one-dimensional p-Laplacian," Computers \& Mathematics with Applications, vol. 48, no. 5-6, pp. 913-925, 2004. 


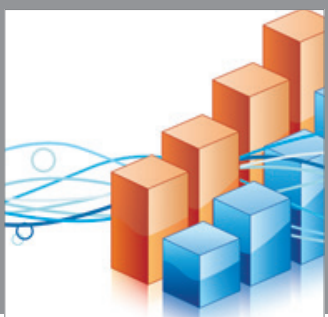

Advances in

Operations Research

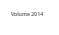

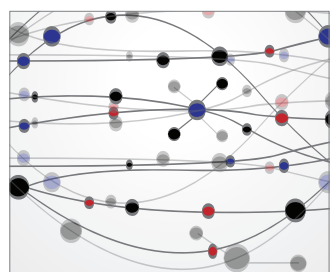

\section{The Scientific} World Journal
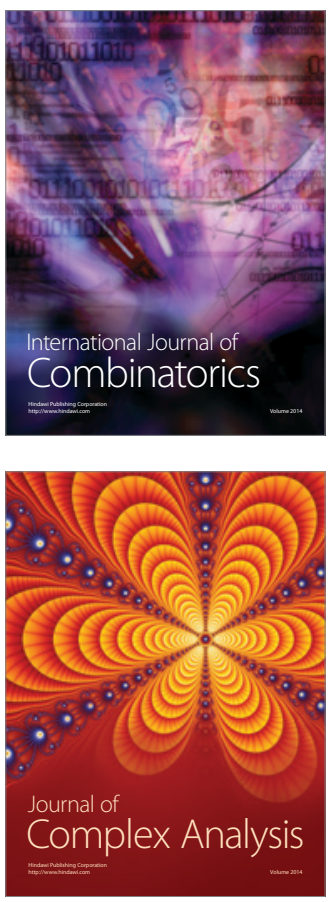

International Journal of

Mathematics and

Mathematical

Sciences
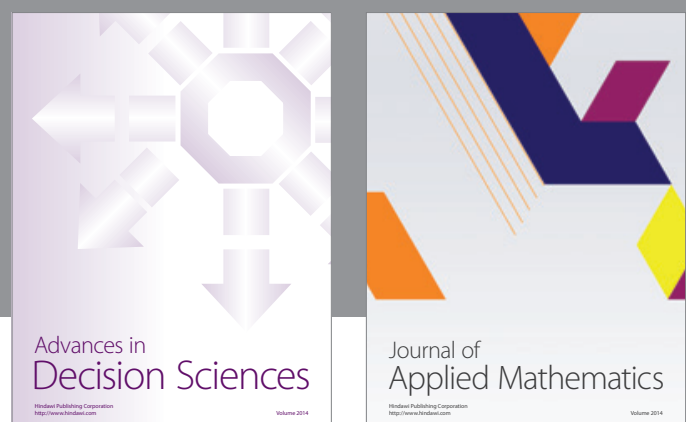

Journal of

Applied Mathematics
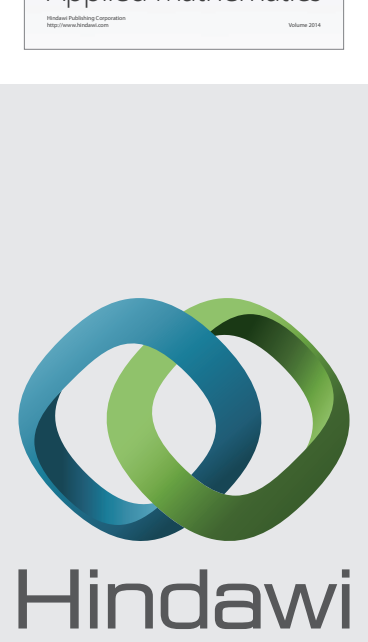

Submit your manuscripts at http://www.hindawi.com
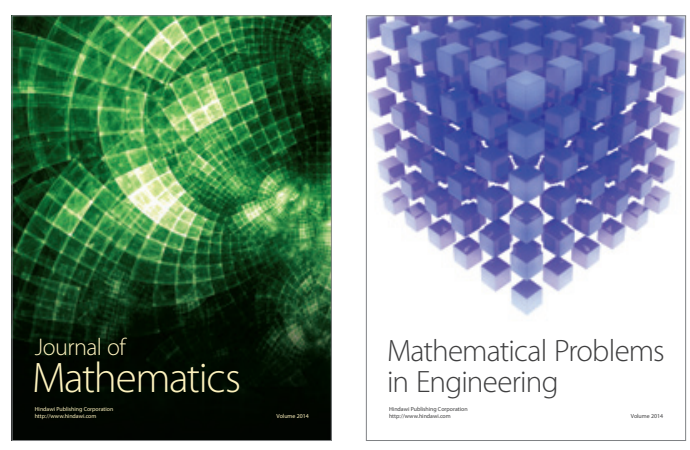

Mathematical Problems in Engineering
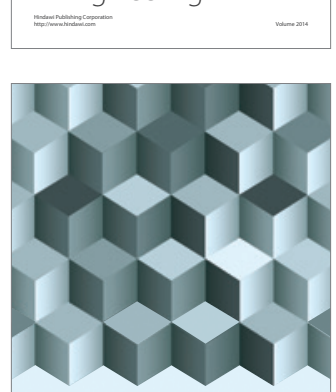

Journal of

Function Spaces
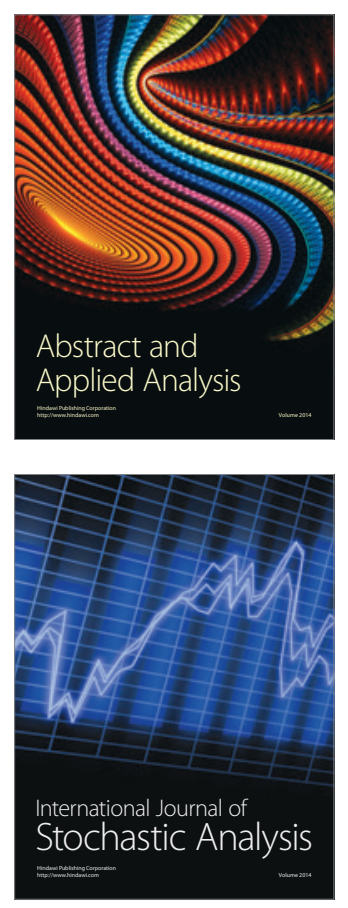

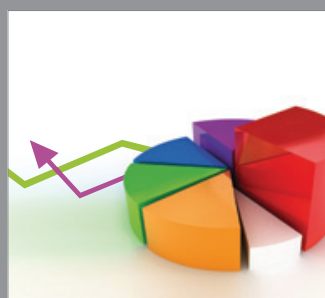

ournal of

Probability and Statistics

Promensencen
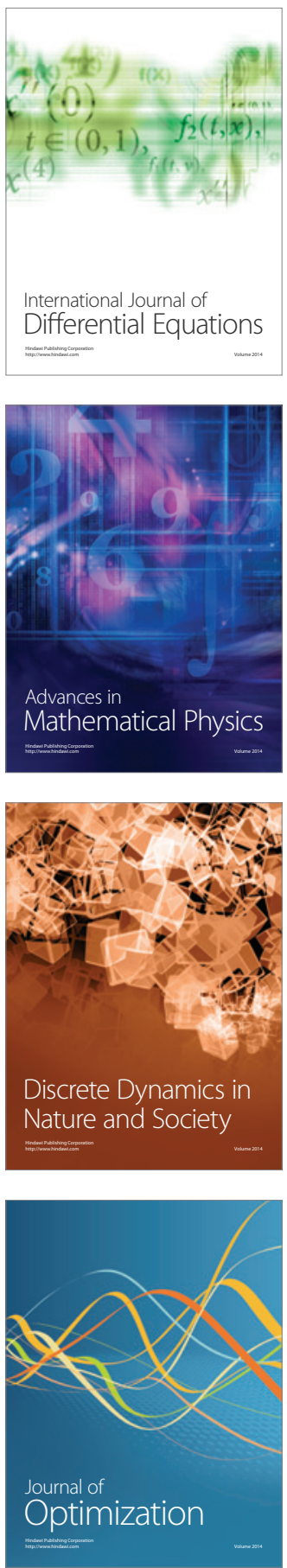